\title{
Social Bonding Decreases the Rewarding Properties of Amphetamine through a Dopamine D1 Receptor-Mediated Mechanism
}

\author{
Yan Liu, ${ }^{1 \star}$ Kimberly A. Young, ${ }^{1 *}$ J. Thomas Curtis, ${ }^{2}$ Brandon J. Aragona, ${ }^{3}$ and Zuoxin Wang ${ }^{1}$ \\ ${ }^{1}$ Department of Psychology, Program in Neuroscience, Florida State University, Tallahassee, Florida 32306, ${ }^{2}$ Department of Pharmacology and Physiology, \\ Center for Health Sciences, Oklahoma State University, Tulsa Oklahoma 74107, and ${ }^{3}$ Department of Psychology, Program in Neuroscience, University of \\ Michigan, Ann Arbor, Michigan 48109
}

\begin{abstract}
Although the protective effects of social bonds on drug use/abuse have been well documented, we know little about the underlying neural mechanisms. Using the prairie vole (Microtus ochrogaster) - a socially monogamous rodent that forms long-term pair bonds after mating - we demonstrate that amphetamine (AMPH) conditioning induced a conditioned place preference (CPP) in sexually naive (SN), but not pair-bonded (PB), males. Although AMPH treatment induced a similar magnitude of dopamine release in the nucleus accumbens (NAcc) of SN and PB males, it had differential effects on NAcc D1 receptor (D1R) binding. Specifically, AMPH treatment increased D1R binding in SN, but decreased D1R binding in PB males. NAcc D1R, but not D2 receptor, antagonism blocked AMPH-induced CPP in SN males and NAcc D1R activation before AMPH conditioning enabled AMPH-induced CPP in PB males. Together, our data demonstrate that pair-bonding experience decreases the rewarding properties of AMPH through a D1R-mediated mechanism.
\end{abstract}

\section{Introduction}

It is well recognized that drug use and abuse have profound consequences on a variety of social behaviors, including social bonding (Young et al., 2011a). Reciprocally, social factors, such as the presence or absence of strong social attachments during development or throughout adulthood, may impact drug use and the vulnerability to drug abuse. For example, strong adolescent-parent attachments have been associated with decreased odds of substance use problems (Ellickson et al., 1999; Bell et al., 2000). Additionally, in individuals who are already addicted, close relationships between spouses aid in recovery from drug addiction (Kosten et al., 1987). In contrast, weak social attachments may increase substance abuse vulnerability as insecure adult attachment styles are positively associated with alcohol and illicit substance abuse (Brennan and Shaver, 1995; Vungkhanching et al., 2004; Caspers et al., 2005).

The neural mechanisms underlying the interaction between social bonding and drug use/abuse are still largely unknown; however, the mesolimbic dopamine (DA) system, particularly DA neurotransmission within the nucleus accumbens (NAcc),

\footnotetext{
Received Feb. 11, 2011; revised April 12, 2011; accepted April 14, 2011.

Author contributions: B.J.A. and Z.W. designed research;Y.L. and J.T.C. performed research; Y.L., K.A.Y., and Z.W. analyzed data; Y.L., K.A.Y., B.J.A., and Z.W. wrote the paper.

This work was supported by National Institutes of Health Grants DAF31-25570 to K.A.Y., HDR01-48462 to J.T.C., and DAR01-19627, DAK02-23048, and MHR01-58616 to Z.X.W. We thank Claudia Lieberwirth, Kelly Lei, Melissa Martin, and Adam Smith for their critical reading of this manuscript. Additionally, we thank Terry E. Robinson for reading an early draft of this manuscript and for providing valuable suggestions.

*Y.L. and K.A.Y. contributed equally to this work.

Correspondence should be addressed to Dr. Zuoxin Wang, Department of Psychology, Florida State University, Tallahassee, FL 32306-1270. E-mail: zwang@psy.fsu.edu.

DOI:10.1523/JNEUROSCI.1006-11.2011

Copyright $\odot 2011$ the authors $\quad 0270-6474 / 11 / 317960-07 \$ 15.00 / 0$
}

may be involved (Young et al., 2011a). Studies in the prairie vole (Microtus ochrogaster) - a socially monogamous rodent that forms enduring bonds between adult mates (i.e., pair bonds) (Insel and Hulihan, 1995; Mattson et al., 2001)—have shown that NAcc DA mediates both the formation and maintenance of pair bonds (Gingrich et al., 2000; Aragona et al., 2003, 2006). Additionally, NAcc DA neurotransmission is activated by all known drugs of abuse (Di Chiara and Imperato, 1988; Bergman et al., 1990; Koob and Nestler, 1997; Nestler, 2005) and repeated psychostimulant drug exposure alters DA release and receptor sensitivity as well as the morphology of NAcc neurons (Henry et al., 1989; Robinson and Kolb, 1999). These changes are thought to underlie drug-induced modifications in behaviors (Robinson and Becker, 1986), including social behaviors (Fiorino and Phillips, 1999).

As both pair-bonding behavior and its regulation by DA neurotransmission within the NAcc have been well characterized in the prairie vole (Gingrich et al., 2000; Aragona et al., 2003, 2006), recent efforts have been made to establish this species as an animal model in which to examine the behavioral interaction between social bonding and drug use/abuse, as well as its underlying neural mechanisms. Amphetamine (AMPH) has been shown to have rewarding properties in prairie voles, as AMPH conditioning induces a conditioned place preference (CPP) in both sexes (Aragona et al., 2007; Liu et al., 2010; Young et al., 2011b); this process is mediated by DA neurotransmission in the NAcc (Curtis and Wang, 2007; Liu et al., 2010). Most interestingly, repeated AMPH exposure inhibits the formation of mating-induced partner preferences in male prairie voles and this AMPH-induced impairment of pair bonding is regulated, at least in part, by NAcc DA D1-type receptor (D1R) activation (Liu et al., 2010). In the 
present study, we investigated the effects of pair-bonding experience on AMPH-induced CPP. We hypothesized that pairbonding experience would decrease the rewarding effects of AMPH and that NAcc DA may be involved in this behavioral phenomenon.

\section{Materials and Methods}

Subjects. Subjects were male prairie voles (M. ochrogaster) from a laboratory breeding colony. Subjects were weaned at $21 \mathrm{~d}$ of age and housed in same-sex sibling pairs in plastic cages $(12 \times 28 \times 16 \mathrm{~cm})$. Water and food were provided ad libitum. All cages were maintained under a 14:10 light: dark cycle and the temperature was kept at $20^{\circ} \mathrm{C}$. Subjects $\sim 75 \mathrm{~d}$ of age were either continuously housed with their same-sex sibling [and thus kept sexually naive $(\mathrm{SN})$ ] or paired with an unrelated intact female for 2 weeks to become pair bonded (PB). Both SN and PB subjects were tested at $\sim 90 \mathrm{~d}$ of age.

$A M P H$ conditioning and CPP testing. These procedures were performed as previously described (Liu et al., 2010; Young et al., 2011b). Briefly, the testing apparatus for CPP consisted of two cages $(12 \times 28 \times$ $16 \mathrm{~cm}$ ), one black with a metal top and one white with a mesh top, joined by a hollow tube $(7.5 \times 16 \mathrm{~cm})$. Although prairie voles generally tend to prefer the white over the dark cage (Aragona et al., 2007), there are a great deal of individual differences in this preference. Therefore, on day 1, we tested all subjects for their initial cage preferences during a $30 \mathrm{~min}$ pretest. During this test, all subjects were allowed free access to both cages and we quantified the amount of time each individual spent in each cage. On days 2-4, subjects received two 40 min conditioning sessions $6 \mathrm{~h}$ apart. In the morning session, subjects received either $1.0 \mathrm{mg} / \mathrm{kg} \mathrm{AMPH}$ (Sigma) dissolved in $0.9 \%$ saline (SN-AMPH and PB-AMPH groups) or saline alone ( $\mathrm{SN}$-saline and $\mathrm{PB}$-saline groups) and were placed in the cage in which they had spent less time during the pretest (conditioned cage). In the afternoon session, all subjects received an injection of saline and were placed in the other cage. On day 5 , subjects were tested again for cage preferences in a $30 \mathrm{~min}$ posttest. Immediately after the posttest, subjects were rapidly decapitated and their brains were frozen on dry ice. Brain sections were subsequently processed for D1R and DA D2-type receptor (D2R) autoradiographic binding.

Brain microdialysis and HPLC-electrochemical detection analysis. Microdialysis probes were constructed as previously described (Curtis and Wang, 2007) and were implanted into the left NAcc (stereotaxic coordinates from bregma: anterior $2.1 \mathrm{~mm}$, lateral $0.6 \mathrm{~mm}$, ventral $6.3 \mathrm{~mm}$ ) under sodium pentabarbitol anesthesia (1 mg/10 kg body weight). Animals were allowed to recover overnight and were then tested the following morning. Probes were perfused continuously at $2.3 \mu \mathrm{l} / \mathrm{min}$ with a solution isotonic for sodium $(144 \mathrm{~mm} \mathrm{NaCl})$, potassium $(2.8 \mathrm{~mm} \mathrm{KCl})$, calcium (1.2 $\mathrm{mm} \mathrm{CaCl}_{2}$ ), and magnesium (0.9 mM $\mathrm{MgCl}_{2}$ ) (Sved and Curtis, 1993).

Following overnight recovery, four $20 \mathrm{~min}$ baseline samples were collected into vials containing $5 \mu l$ of $0.1 \mathrm{~N}$ perchloric acid. Thereafter, subjects received an intraperitoneal injection of AMPH $(1.0 \mathrm{mg} / \mathrm{kg})$ and dialysate samples were continuously collected at $20 \mathrm{~min}$ intervals for $3 \mathrm{~h}$. Dialysate samples were immediately frozen at $-80^{\circ} \mathrm{C}$ until analyzed. The amounts of DA and DOPAC in each sample were determined using HPLC with electrochemical detection (ECD), as described previously (Curtis and Wang, 2007). At the end of the sampling period, subjects were killed to assess probe placement.

$D A$ receptor autoradiography. Coronal brain sections $(20 \mu \mathrm{m})$ at 120 $\mu \mathrm{m}$ intervals were processed for DA receptor autoradiographic binding using an established method (Aragona et al., 2006). Briefly, sections were rinsed in $50 \mathrm{~mm}$ Tris- $\mathrm{HCl}, \mathrm{pH} 7.4$, and incubated in $50 \mathrm{~mm}$ Tris- $\mathrm{HCl}$ ion buffer containing $120 \mathrm{~mm} \mathrm{NaCl}, 5 \mathrm{~mm} \mathrm{KCl}, 2 \mathrm{mM} \mathrm{CaCl}_{2}$, and $1 \mathrm{mM} \mathrm{MgCl}_{2}$ with either $\left[{ }^{125} \mathrm{I}\right] \mathrm{SCH} 23982$ (D1R ligand) or $\left[{ }^{125} \mathrm{I}\right] 2$ '-iodospiperone (D2R ligand) (PerkinElmer). Thereafter, sections were fixed in $0.1 \%$ paraformaldehyde and rinsed thoroughly in Tris- $\mathrm{HCl}$ ion buffer. Slides were dipped in distilled water, blown dry, and exposed to BioMax MR film (Kodak) to generate autoradiograms. The optical densities of D1R and D2R binding in the NAcc and caudate putamen $(\mathrm{CP})$ were quantified in three anatomically matched brain sections per animal from autoradiograms using a computerized image program (NIH Image 1.64).

Stereotaxic cannulation and microinjection. Subjects were anesthetized with sodium pentobarbital and 26-gauge bilateral stainless steel cannulae (Plastics One) were stereotaxically implanted and aimed at the NAcc, as described previously (Aragona et al., 2003). Subjects were allowed to recover for 3-7 d. On each of the $3 \mathrm{~d}$ of conditioning, 30 min before AMPH injections, subjects received microinjections of either artificial CSF (200 nl/side) or CSF containing the D1R agonist SKF 38393, the D1R antagonist SCH 23390, or the D2R antagonist eticlopride (Sigma). After CPP testing, all subjects were rapidly decapitated and their brains were extracted to verify injection sites histologically. Subjects with misplaced cannulae were excluded from data analysis.

Data quantification and statistical analysis. CPP was determined by a paired-samples $t$ test comparing the amounts of time that subjects spent in the conditioned cage between pretests and posttests. Cage entries between pretests and posttests were also analyzed by a $t$ test to assess whether AMPH, a D1R agonist, D1R, or a D2R antagonist affected locomotor activity. Absolute amounts of baseline DA and DOPAC in dialysates were compared between groups using a $t$ test. For assessment of AMPH effects across time, the amounts of DA and DOPAC in each baseline and post-AMPH sample were expressed as a percentage of the mean baseline amount. These values then were analyzed by repeatedmeasures ANOVA followed by a Student-Neuman-Keuls (SNK) post hoc test. Finally, group differences in the densities of D1R and D2R binding in the NAcc and CP were analyzed by a two-way ANOVA followed by a SNK post hoc test.

Experimental design. Experiment 1 was designed to reveal the effects of pair-bonding experience on AMPH-induced CPP. SN and PB males were pretested in the CPP apparatus. They were then divided into four groups that received injections of saline ( $n=5$ for SN males and $n=9$ for PB males) or AMPH ( $1.0 \mathrm{mg} / \mathrm{kg} ; n=8$ for SN males and $n=8$ for PB males) during morning conditioning sessions over the next $3 \mathrm{~d}$ (Liu et al., 2010). Thereafter, all subjects received a CPP posttest.

Experiment 2 compared AMPH-induced DA release in the NAcc between SN $(n=6)$ and PB $(n=5)$ males. Subjects were implanted with a microdialysis probe aimed at the NAcc. After overnight recovery with continuous perfusion of an isotonic solution through the probes, four 20 min baseline dialysate samples were collected. Thereafter, subjects received an intraperitoneal injection of AMPH $(1.0 \mathrm{mg} / \mathrm{kg})$ and dialysate samples were continuously collected every $20 \mathrm{~min}$ for $3 \mathrm{~h}$. These samples were subsequently analyzed for DA and DOPAC concentrations using HPLC-ECD analysis (Curtis and Wang, 2007).

Experiment 3 examined the effect of interactions between pair bonding and AMPH treatment on DA receptor binding in the NAcc. Brain sections from the subjects tested in experiment 1 were processed for D1R and D2R binding using receptor autoradiography.

Experiment 4 tested the role of NAcc DA receptors in AMPH-induced CPP. SN males were implanted with guide cannulae bilaterally aimed at the NAcc. After $3 \mathrm{~d}$ of recovery, subjects received a CPP pretest and were then randomly assigned into one of five experimental groups [intraNAcc injections of CSF $(n=8)$ or intra-NAcc injections of CSF containing a low ( $4 \mathrm{ng} / \mathrm{side} ; n=8)$ or high $(100 \mathrm{ng} / \mathrm{side} ; n=6)$ dose of a D1R antagonist, SCH 23390, or a low ( $4 \mathrm{ng} /$ side; $n=8$ ) or high (100 ng/side; $n=7$ ) dose of a D2R antagonist, eticlopride]. Thirty minutes later, subjects received an injection of AMPH ( $1.0 \mathrm{mg} / \mathrm{kg}$, i.p.). This procedure was repeated for 3 consecutive days during AMPH conditioning. Thereafter, subjects received a CPP posttest.

Experiment 5 examined the role of NAcc D1Rs in mediating AMPHinduced $\mathrm{CPP}$ in $\mathrm{PB}$ males. $\mathrm{PB}$ subjects were divided into three groups, receiving intra-NAcc injections of CSF $(n=10)$ or CSF containing a D1R agonist, SKF $38393(0.4 \mathrm{ng} /$ side; $n=12)$, or a D1R antagonist, SCH 23390 ( $4 \mathrm{ng} / \mathrm{side} ; n=10$ ), before AMPH conditioning. Brain cannulation, AMPH injections, and CPP tests were the same as described in experiment 4 . 

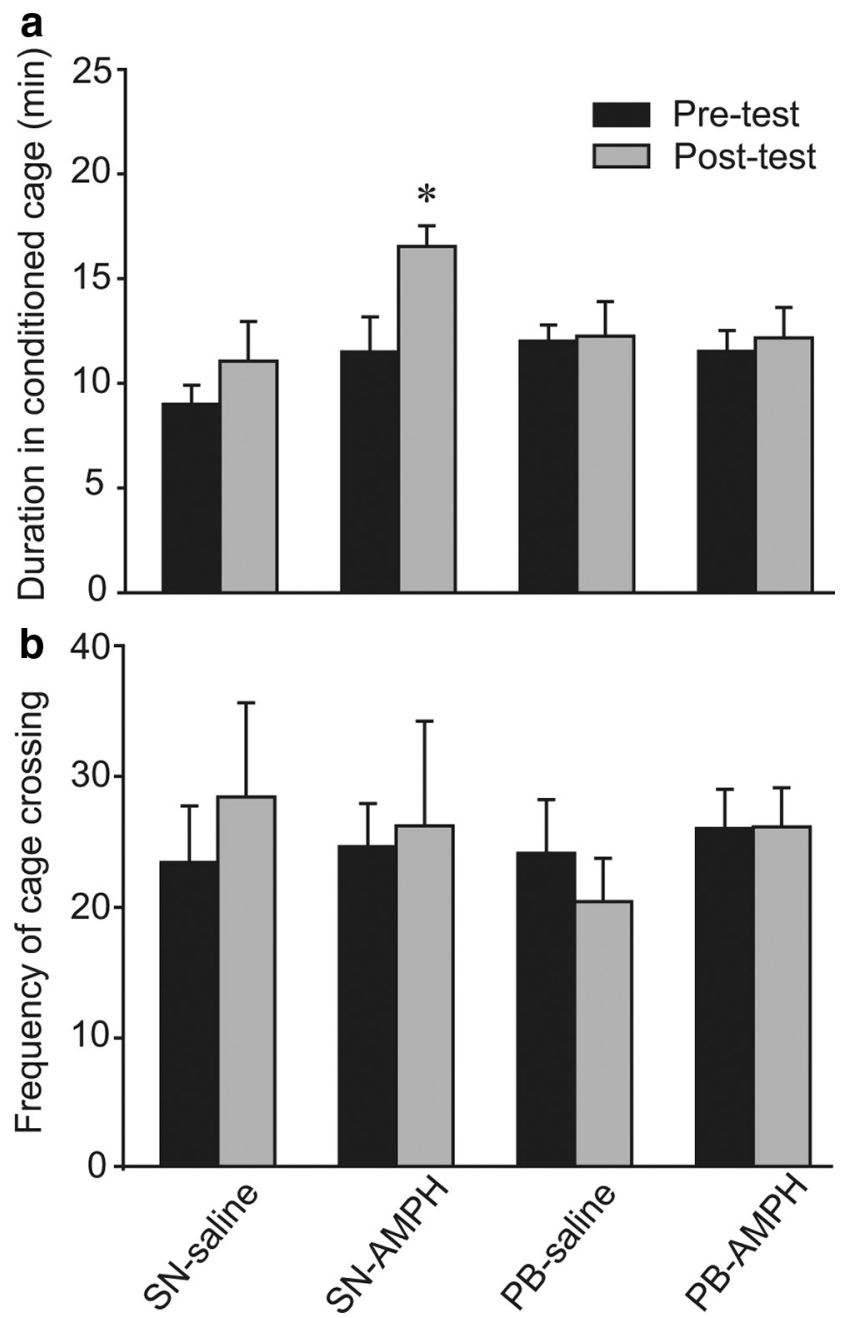

Figure 1. AMPH conditioning induces a $C P P$ in $S N$ but not $P B$ male prairie voles. $\boldsymbol{a}, S N$ or $P B$ males that received saline (SN-saline or PB-saline, respectively) during $3 \mathrm{~d}$ of conditioning did not form a CPP, i.e., they spent equal amounts of time in the conditioned cage during the $30 \mathrm{~min}$ pretests and posttests. AMPH conditioning induced a CPP in SN males (SN-AMPH), but not in PB males (PB-AMPH), indicating that pair-bonding experience may decrease the rewarding properties of AMPH. $\boldsymbol{b}$, No differences in the frequency of cage crossing between pretests and posttests were noted in any group. ${ }^{*} p<0.05$. Error bars indicate SEM.

\section{Results}

\section{Pair-bonding experience decreases the rewarding properties} of AMPH

In our previous study, AMPH treatment impaired matinginduced partner preferences in male prairie voles, indicating an inhibitory effect of AMPH exposure on pair-bonding behavior (Liu et al., 2010). In the present study, we tested the reciprocal relationship: the effects of pair bonding experience on AMPH reward. Three days of conditioning with $1.0 \mathrm{mg} / \mathrm{kg}$ AMPH induced a CPP in SN males $(t=2.45, p<0.05)$ but not in males that had been paired with a female for 2 weeks (i.e., PB males) (Fig. 1a). Saline injections had no effect in either group. Importantly, no differences were found in the frequencies of the animals' cage crossings between pretests and posttests, suggesting that impaired CPP in PB males was not due to altered locomotor activity during the behavioral test (Fig. 1b).

\section{AMPH treatment induces DA release in the NAcc in both $\mathrm{SN}$ and $P B$ males}

There were no significant differences between $\mathrm{SN}$ and $\mathrm{PB}$ males in the absolute amounts of DA or DOPAC in microdialysis baseline

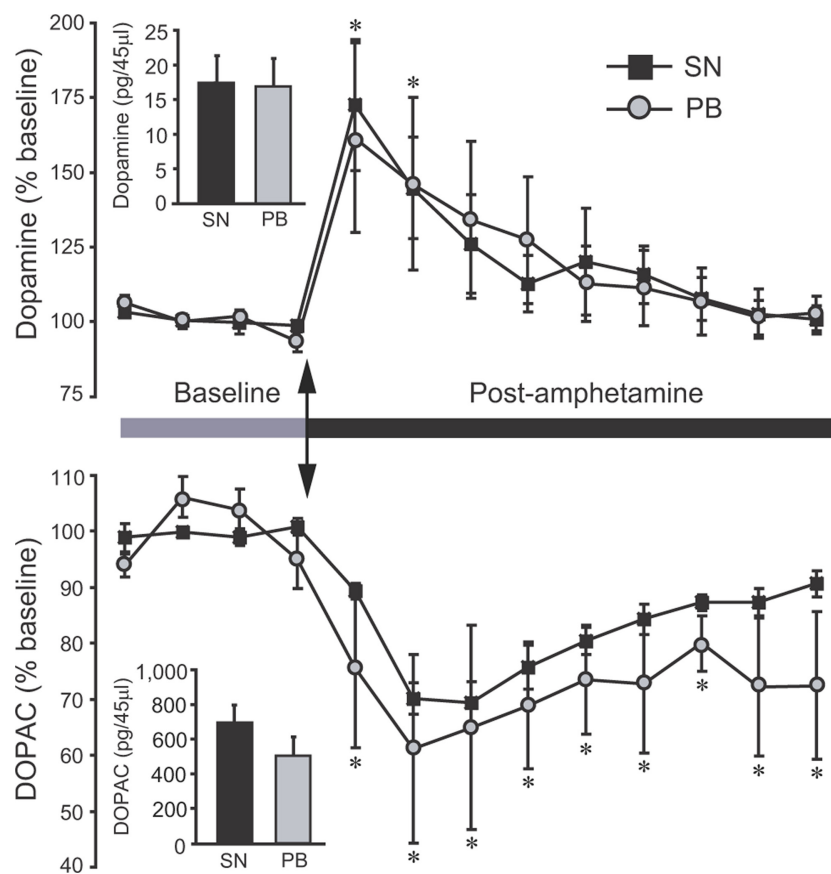

Figure 2. Levels of extracellular DA and DOPAC in the NAcc of SN and PB males following amphetamine treatment. Absolute amounts of DA and DOPAC in baseline dialysates did not differ between SN and PB males (insets). Peripheral amphetamine administration increased extracellular DA and decreased extracellular DOPAC within the NAcc. The magnitude of these responses was not different between SN and PB males. Arrows indicate times of injection. *Significant difference from the within-social experience baseline. Error bars indicate SEM.

samples (Fig. 2, insets). AMPH administration produced significant increases in extracellular DA $\left(F_{(12,108)}=8.42, p<0.001\right)$. However, the magnitude and duration of these increases did not differ between SN and PB males-DA levels were significantly higher than baseline in both groups for each of the first two sampling periods ( $40 \mathrm{~min}$ total) and then slowly returned to baseline (Fig. 2, top). AMPH administration significantly decreased extracellular DOPAC in the NAcc in both SN and PB males $\left(F_{(12,108)}=13.54, p<0.001\right)$ and, again, these effects were similar in both groups. Neither SN nor PB males regained baseline levels before the end of sampling (Fig. 2, bottom).

\section{AMPH treatment differentially alters D1R binding in the NAcc of SN and PB males}

Previous studies have demonstrated that AMPH treatment enhances NAcc D1R gene and protein expression (Liu et al., 2010). Additionally, pair-bonding experience elevates D1R binding (Aragona et al., 2006) in the NAcc of male prairie voles. Therefore, we hypothesized that alterations in DA receptor binding in the NAcc may underlie the behavioral interaction between pair bonding and AMPH reward. We processed brain sections from subjects used in the CPP tests for DA receptor autoradiographic binding. Two-way ANOVA analysis indicated a significant interaction between social experience ( $\mathrm{SN}$ or $\mathrm{PB}$ ) and injection type (saline or AMPH) on D1R binding in the $\operatorname{NAcc}\left(F_{(1,29)}=17.63\right.$, $p<0.01)$. The post hoc test revealed that the densities of D1R binding in the NAcc of SN-AMPH and PB-saline groups were comparable and significantly higher than those of SN-saline and PB-AMPH groups (Fig. 3a). Neither AMPH treatment nor pairbonding experience altered the density of D2R binding in the NAcc (Fig. 3b). In addition, no group differences were found in 

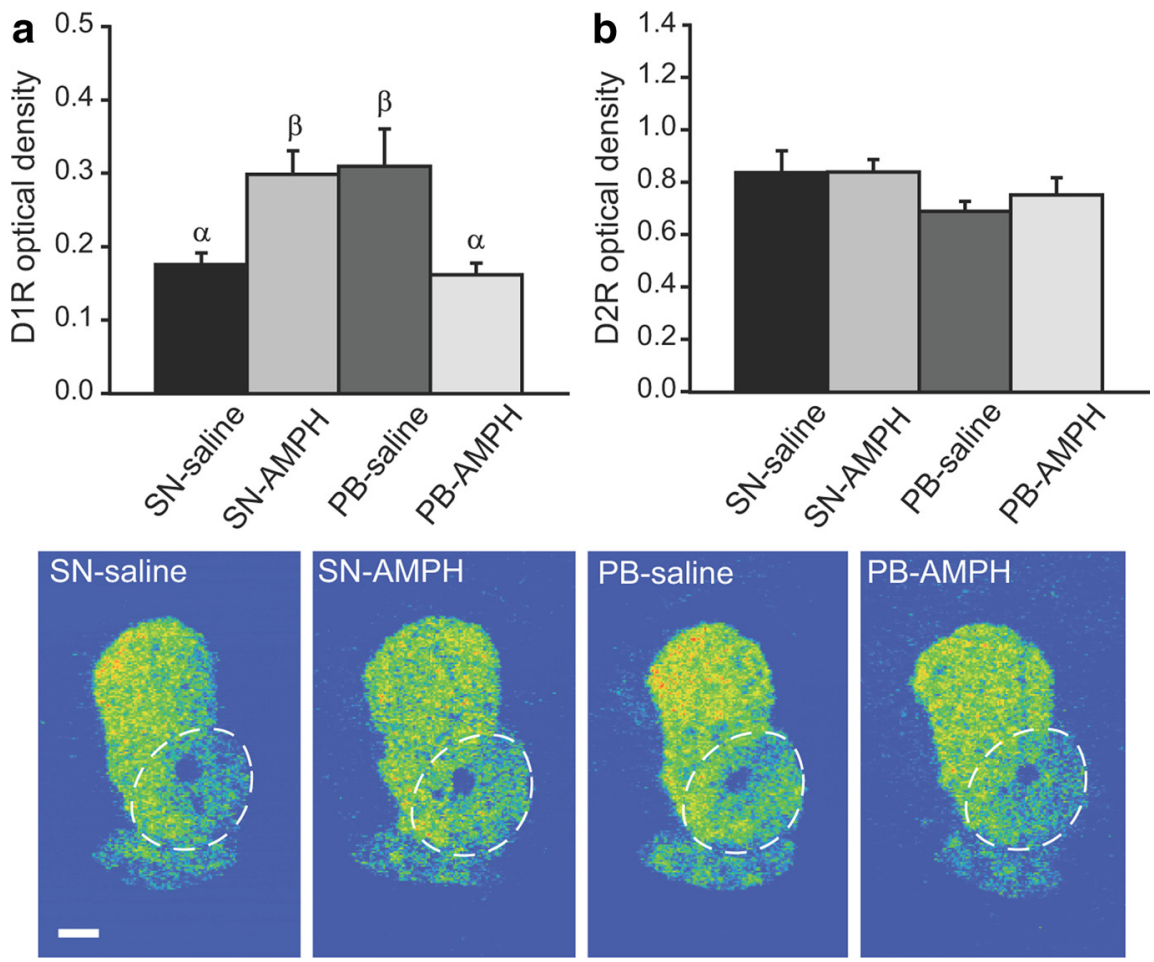

Figure 3. Pair bonding and AMPH interact to affect DA receptor binding. $\boldsymbol{a}, \mathrm{AMPH}$ conditioning significantly increased the density of NAcc D1R binding in SN males (SN-AMPH) compared with saline-injected controls (SN-saline). However, PB males injected with saline (PB-saline) showed a level of D1R binding comparable to that of SN-AMPH males, and AMPH conditioning significantly decreased the density of NAcc D1R binding (PB-AMPH). $\boldsymbol{b}$, No group differences were found in NAcc D2R binding. Photomicrographs illustrate D1R binding in the NAcc (circled) in the prairie vole brain. Bars with different Greek letters differ significantly from each other. Error bars indicate SEM. Scale bar, $1 \mathrm{~mm}$

either D1R or D2R binding in the caudate-putamen (data are not shown)

\section{NAcc D1R activation mediates AMPH reward in $\mathrm{SN}$ males}

In male prairie voles, subcutaneous injections of a D1R, but not D2R, antagonist before AMPH conditioning sessions eliminated AMPH-induced CPP (Liu et al., 2010). Given the established role of NAcc DA in AMPH reward in other rodent species (Yokel and Wise, 1975; Kehoe et al., 1996), we hypothesized that access to D1Rs in the NAcc during conditioning is essential for AMPHinduced CPP in SN male prairie voles. We found that SN males that received CSF injections into the NAcc displayed AMPHinduced CPP $(t=2.90, p<0.01)$ (Fig. 4). However, intra-NAcc administration of a D1R antagonist, SCH 23390, at either a low (4 $\mathrm{ng} /$ side) or high (100 ng/side) dose before conditioning sessions eliminated AMPH-induced CPP (Fig. 4). In contrast, intra-NAcc administration of a D2R antagonist, eticlopride, at either a low (4 $\mathrm{ng} /$ side; $t=3.25, p<0.01)$ or high $(100 \mathrm{ng} / \mathrm{side} ; t=2.30, p<$ 0.05 ) dose did not block AMPH-induced CPP (Fig. 4). No differences were found in the frequencies of cage crosses between the pretests and posttests in any group, indicating no effect of treatment on locomotor activity (data are not shown).

\section{Activation of D1Rs in the NAcc enables AMPH-induced CPP in PB males}

Previous studies have shown that NAcc D1R activation is essential for AMPH-induced CPP and selective aggression, and that it impairs partner preference formation in male prairie voles (Aragona et al., 2006; Liu et al., 2010). Given the role of D1Rs in those behaviors and the finding that NAcc D1R binding is lower in
PB-AMPH males than in PB-saline and SN-AMPH males (Fig. 3a), we hypothesized that decreased D1R activity in the NAcc may be responsible for the lack of $\mathrm{AMPH}$-induced CPP in PB males. To test this hypothesis, we injected CSF or CSF containing a D1R agonist or antagonist site-specifically into the NAcc before each of the three conditioning sessions and then tested for the presence of AMPHinduced CPP. As expected, $\mathrm{PB}$ males that received CSF injections did not show AMPH-induced CPP (Fig. 5). However, $\mathrm{PB}$ males that received intra-NAcc injections of the D1R agonist $(t=4.69, p<$ 0.001 ), but not antagonist, displayed AMPH-induced CPP (Fig. 5). There were no differences in the frequencies of cage crosses between the pretest and posttest for any group (data are not shown).

\section{Discussion}

Studies in both humans and animal models have demonstrated a strong relationship between drug use/abuse and social behavior (Young et al., 2011a). Because of its well known role in the generation of motivated behaviors, the mesolimbic DA system is in a key position to mediate the interaction between drugs of abuse and social behavior. We have recently demonstrated that repeated exposure to AMPH impairs pair-bond formation in male prairie voles and that NAcc DA mediates this effect (Liu et al., 2010). In the present study, we demonstrate that pair-bonding experience impairs AMPH-induced CPP and that this effect is also mediated by NAcc DA. Together, these studies demonstrate a reciprocal interaction between pair bonding and AMPH reward and suggest a role for NAcc DA in regulating such interactions.

In the current study, we used a previously established CPP paradigm (Liu et al., 2010; Young et al., 2011b) to investigate the effects of pair-bonding experience on the rewarding properties of AMPH. We use the ambiguous term "rewarding properties" to describe the impact of AMPH on place conditioning because it allows us to simultaneously address the individual components of reward-including hedonics, associative learning, and incentive motivation (Berridge and Robinson, 2003) — that have been implicated in processes underlying place conditioning (Hnasko et al., 2005; White et al., 2005; Cunningham and Patel, 2007), without distinguishing between them. Our results demonstrate that $\mathrm{AMPH}$ conditioning induced a CPP in SN, but not PB, male voles, and as such, offer the first empirical evidence that pairbonding experience decreases the rewarding properties of AMPH. Although this is the only study to investigate the effects of pairbonding experience on the rewarding properties of drugs of abuse, previous studies have demonstrated that other social experiences/ factors may also influence drug reward. For example, rats reared with multiple social cohorts self-administered less AMPH (Bardo et al., 2001) and cocaine (Schenk et al., 1987) over repeated sessions than rats reared alone. Similarly, rats reared in an enriched environment that contained novel objects and social cohorts selfadministered less AMPH, extinguished self-administration behavior 


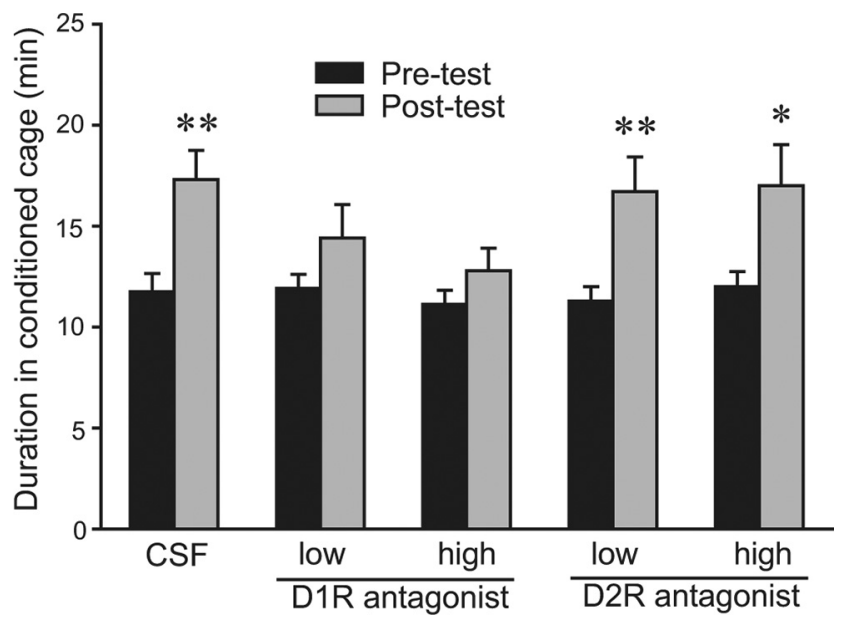

Figure 4. The involvement of $\mathrm{NAcC} D A \mathrm{D} 1 \mathrm{R}$ and D2R in AMPH-induced CPP in sexually naive male prairie voles. All subjects received AMPH during conditioning sessions. On each of the $3 \mathrm{~d}$ of conditioning, 30 min before AMPH injections, subjects received bilateral intra-NAcc microinjections of either artificial CSF or CSF containing a low or high dose of SCH 23390 (D1R antagonist) or eticlopride (D2R antagonist). Subjects treated with CSF and either dose of the D2R antagonist formed an AMPH-induced CPP, i.e., they spent significantly more time in the conditioned cage during the posttest than during the pretest. However, treatment with either dose of the D1R antagonist eliminated AMPH-induced CPP, indicating that NAcc D1R, but not D2R, activation is essential for AMPH reinforcement in sexually naive male prairie voles. ${ }^{*} p<0.05$, ${ }^{* *} p<0.01$. Error bars indicate SEM.

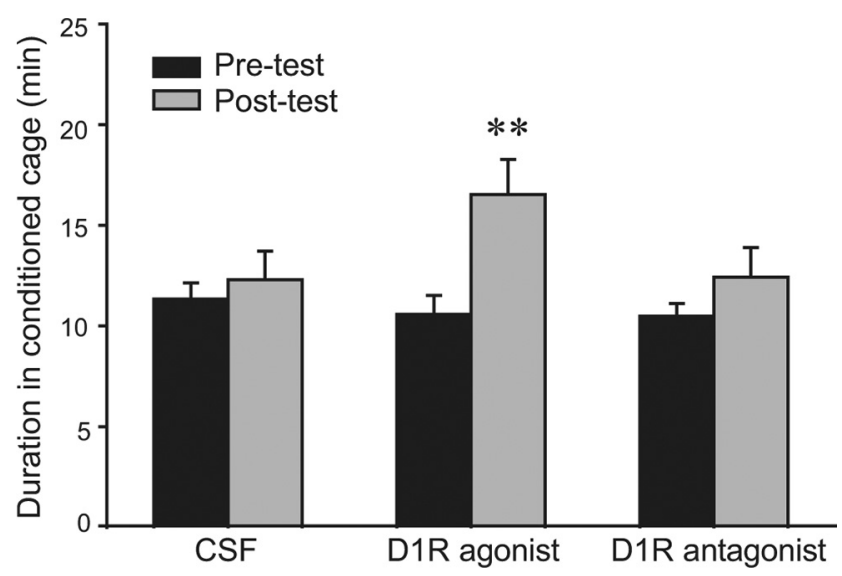

Figure 5. Activation of the DA D1R in the NAcc enables AMPH-induced CPP in pair-bonded male prairie voles. All subjects were pair bonded and received AMPH during conditioning sessions. On each of the $3 \mathrm{~d}$ of conditioning, $30 \mathrm{~min}$ before AMPH injections, subjects received bilateral intra-NAcc injections of either CSF or CSF containing SFK 38393 (D1R agonist, 0.4 $\mathrm{ng} /$ side) or SCH 23390 (D1R antagonist, $4 \mathrm{ng} /$ side). Subjects treated with (SF or a D1R antagonist did not form a CPP. However, activation of D1Rs with a D1R agonist induced CPP in pairbonded male prairie voles. ${ }^{* *} p<0.001$. Error bars indicate SEM.

sooner, and required higher doses of $\mathrm{AMPH}$ to reinstate drugseeking than did rats housed alone (Schenk et al., 1988; Bardo et al., 1995, 2001; Green et al., 2002; Stairs et al., 2006). Furthermore, female rats trained to lever-press for cocaine showed markedly fewer responses after becoming pregnant and following parturition (Hecht et al., 1999), suggesting that the reproductive process and neurobiological changes involved with maternal experience may decrease the reinforcing properties of cocaine. This notion is further supported by the finding that virgin rats preferred an environment associated with cocaine and readily expressed a cocaine-induced CPP (Seip et al., 2008), whereas lactating dams strongly preferred an environment associated with pups over one associated with cocaine (Mattson et al., 2001). Together, these studies imply that social fac- tors may reduce the rewarding properties of psychostimulants. Our results extend these findings and demonstrate that pair-bonding experience impairs AMPH reward in prairie voles.

The rewarding properties of psychostimulant drugs of abuse, such as AMPH, are dependent on drug-induced increases in NAcc DA release and the subsequent activation of DA receptors (Yokel and Wise, 1975; Di Chiara and Imperato, 1988; Bergman et al., 1990; Kehoe et al., 1996). Therefore, alterations in either of these factors may underlie the effects of pair-bonding experience on AMPH reward. Levels of released DA have been closely correlated with the positive subjective effects of drugs of abuse (Volkow et al., 1999; Drevets et al., 2001; Leyton, 2010) and psychostimulant-induced NAcc DA release has been altered by social experience in some cases [e.g., isolation early in life (Kehoe et al., 1996; Kosten et al., 2005)], but not others [e.g., enriched social housing (Bardo et al., 1995)]. In the present study, AMPH treatment increased the level of extracellular DA in the NAcc, which is consistent with previous reports (Di Chiara and Imperato, 1988; Curtis and Wang, 2007; McKittrick and Abercrombie, 2007). Furthermore, AMPH induced an immediate and prolonged decrease in the level of extracellular DOPAC in both groups. This finding is consistent with those of previous studies and the known role of AMPH in the inhibition of monoamine oxidase-an enzyme involved in the degradation of DA (Green and el Hait, 1978; Jones et al., 1998; Curtis and Wang, 2007). In both cases, the magnitude and temporal pattern of extracellular changes in the NAcc were comparable between SN and $\mathrm{PB}$ males, suggesting that it is unlikely that the effects of pair bonding on AMPH reward are related to the release or metabolism of NAcc DA.

Although pair-bonding experience did not influence AMPHinduced DA release or metabolism, it did influence the effects of AMPH on NAcc DA receptor binding (i.e., DA receptor expression and/or affinity). For example, consistent with previous studies, AMPH treatment increased NAcc D1R expression in SN males (Liu et al., 2010) and pair-bonding experience also elevated NAcc D1R binding (Aragona et al., 2006). However, the effect of AMPH on NAcc D1R binding was reversed in PB males compared with $\mathrm{SN}$ males, as $\mathrm{PB}$ males showed a significant decrease in D1R binding following AMPH treatment. No group differences were found in NAcc D2R binding in either the current or previous studies (Aragona et al., 2006; Liu et al., 2010). Together, these data demonstrate that pair-bonding experience and AMPH exposure each leads to D1R-specific changes within the NAcc of SN males. Further, as AMPH oppositely affected NAcc D1R binding in $\mathrm{SN}$ and $\mathrm{PB}$ voles, our data indicate that social bonding may be an important mediating factor in the effects of AMPH on the mesolimbic DA system. Correspondingly, cocaine has been found to induce a robust positive blood oxygenation leveldependent (BOLD) signal change throughout the mesolimbic DA system in virgin females, but a largely negative BOLD response in lactating dams, as measured by functional magnetic resonance imaging (Ferris et al., 2005), thus further indicating that social/sexual experience may play an important role in the neurobiological response to drugs of abuse. Alterations in receptor binding, such as those described above, may have profound effects on behavior because they modify the brain's responsiveness to released neurotransmitters. In $\mathrm{SN}$ males, for example, AMPH-induced increases in D1R binding may play an important role during AMPH conditioning as intra-NAcc blockade of D1R, but not D2R, inhibited AMPH-induced CPP-a finding consistent with those in other species (Baker et al., 1998; Pitchers et al., 2010). Consequently, in PB males, decreased NAcc D1R binding 
may underlie the lack of an AMPH-induced CPP as NAcc D1R activation during AMPH conditioning enabled AMPH-induced $\mathrm{CPP}$ in PB males. Collectively, these data indicate that AMPHinduced decreases in NAcc D1R expression/affinity may underlie the effects of pair-bonding experience on AMPH reward.

Two interesting parallels between our findings and those from other studies that have investigated the relationship between drugs of abuse and social behavior are worth mentioning. First, pair bonding and repeated AMPH exposure each independently resulted in similar changes in the mesolimbic DA system of male prairie voles [i.e., enhanced D1R binding (Aragona et al., 2006) and expression (Liu et al., 2010) in the NAcc]. Similarly, sexual experience and repeated exposure to psychostimulants each increased the density of dendritic spines on medium spiny neurons within the NAcc shell in rats (Robinson and Kolb, 1999; Pitchers et al., 2010). Furthermore, pup suckling in lactating dams and cocaine administration in virgin females induced similar patterns of positive BOLD activation in the mesocorticolimbic system (Ferris et al., 2005). Together, these data support the notion that drugs of abuse usurp neural mechanisms and circuits that mediate adaptive behaviors (Panksepp et al., 2002). Second, our data suggest that the differential neurobiological responses to AMPH in $\mathrm{SN}$ and $\mathrm{PB}$ males may underlie group differences in AMPHinduced behavior. Similarly, lactating rats showed a suppression of activity within the mesocorticolimbic DA system in response to cocaine (as opposed to the increase in activity noted in virgin females) (Ferris et al., 2005) and a reduction in cocaine selfadministration (Hecht et al., 1999), further supporting the notion that physiological changes associated with social experience may attenuate the rewarding properties of drugs of abuse.

The presence of strong social bonds in adulthood may decrease the vulnerability to drug abuse (Kosten et al., 1987). However, the neural mechanisms underlying this behavioral phenomenon are relatively unknown, perhaps due to the lack of an appropriate animal model. In the present study, we established the prairie vole as an animal model to investigate the neural mechanisms underlying the protective effects of adult social bonding on the vulnerability to drug abuse. Our findings indicate that pair-bonding experience decreases the rewarding properties of AMPH and that the mesolimbic DA system, particularly DA neurotransmission in the NAcc, mediates this effect. These findings, together with those from our previous study (Liu et al., 2010), establish a foundation for future investigation of the neural mechanisms underlying the reciprocal relationship between drug use/abuse and social bonding, which may ultimately provide important insight into the prevention or treatment of drug abuse.

\section{References}

Aragona BJ, Liu Y, Curtis JT, Stephan FK, Wang Z (2003) A critical role for nucleus accumbens dopamine in partner-preference formation in male prairie voles. J Neurosci 23:3483-3490.

Aragona BJ, Liu Y, Yu YJ, Curtis JT, Detwiler JM, Insel TR, Wang Z (2006) Nucleus accumbens dopamine differentially mediates the formation and maintenance of monogamous pair bonds. Nat Neurosci 9:133-139.

Aragona BJ, Detwiler JM, Wang Z (2007) Amphetamine reward in the monogamous prairie vole. Neurosci Lett 418:190-194.

Baker DA, Fuchs RA, Specio SE, Khroyan TV, Neisewander JL (1998) Effects of intraaccumbens administration of SCH-23390 on cocaineinduced locomotion and conditioned place preference. Synapse 30:181-193.

Bardo MT, Bowling SL, Rowlett JK, Manderscheid P, Buxton ST, Dwoskin LP (1995) Environmental enrichment attenuates locomotor sensitization, but not in vitro dopamine release, induced by amphetamine. Pharmacol Biochem Behav 51:397-405.
Bardo MT, Klebaur JE, Valone JM, Deaton C (2001) Environmental enrichment decreases intravenous self-administration of amphetamine in female and male rats. Psychopharmacology (Berl) 155:278-284.

Bell NJ, Forthun LF, Sun SW (2000) Attachment, adolescent competencies, and substance use: developmental considerations in the study of risk behaviors. Subst Use Misuse 35:1177-1206.

Bergman J, Kamien JB, Spealman RD (1990) Antagonism of cocaine selfadministration by selective dopamine $\mathrm{D}(1)$ and $\mathrm{D}(2)$ antagonists. Behav Pharmacol 1:355-363.

Berridge KC, Robinson TE (2003) Parsing reward. Trends Neurosci 26:507-513.

Brennan KA, Shaver PR (1995) Dimensions of adult attachment, affect regulation, and romantic relationship functioning. Pers Soc Psychol Bull 21:267-283.

Caspers KM, Cadoret RJ, Langbehn D, Yucuis R, Troutman B (2005) Contributions of attachment style and perceived social support to lifetime use of illicit substances. Addict Behav 30:1007-1011.

Cunningham CL, Patel P (2007) Rapid induction of Pavlovian approach to an ethanol-paired visual cue in mice. Psychopharmacology (Berl) 192:231-241.

Curtis JT, Wang Z (2007) Amphetamine effects in microtine rodents: a comparative study using monogamous and promiscuous vole species. Neuroscience 148:857-866.

Di Chiara G, Imperato A (1988) Drugs abused by humans preferentially increase synaptic dopamine concentrations in the mesolimbic system of freely moving rats. Proc Natl Acad Sci U S A 85:5274-5278.

Drevets WC, Gautier C, Price JC, Kupfer DJ, Kinahan PE, Grace AA, Price JL, Mathis CA (2001) Amphetamine-induced dopamine release in human ventral striatum correlates with euphoria. Biol Psychiatry 49:81-96.

Ellickson PL, Collins RL, Bell RM (1999) Adolescent use of illicit drugs other than marijuana: how important is social bonding and for which ethnic groups? Subst Use Misuse 34:317-346.

Ferris CF, Kulkarni P, Sullivan JM Jr, Harder JA, Messenger TL, Febo M (2005) Pup suckling is more rewarding than cocaine: evidence from functional magnetic resonance imaging and three-dimensional computational analysis. J Neurosci 25:149-156.

Fiorino DF, Phillips AG (1999) Facilitation of sexual behavior and enhanced dopamine efflux in the nucleus accumbens of male rats after D-amphetamine-induced behavioral sensitization. J Neurosci 19:456-463.

Gingrich B, Liu Y, Cascio C, Wang Z, Insel TR (2000) Dopamine D2 receptors in the nucleus accumbens are important for social attachment in female prairie voles (Microtus ochrogaster). Behav Neurosci 114:173-183.

Green AL, el Hait MA (1978) Inhibition of mouse brain monoamine oxidase by (+)-amphetamine in vivo. J Pharm Pharmacol 30:262-263.

Green TA, Gehrke BJ, Bardo MT (2002) Environmental enrichment decreases intravenous amphetamine self-administration in rats: dose-response functions for fixed- and progressive-ratio schedules. Psychopharmacology (Berl) 162:373-378.

Hecht GS, Spear NE, Spear LP (1999) Changes in progressive ratio responding for intravenous cocaine throughout the reproductive process in female rats. Dev Psychobiol 35:136-145.

Henry DJ, Greene MA, White FJ (1989) Electrophysiological effects of cocaine in the mesoaccumbens dopamine system: repeated administration. J Pharmacol Exp Ther 251:833-839.

Hnasko TS, Sotak BN, Palmiter RD (2005) Morphine reward in dopaminedeficient mice. Nature 438:854-857.

Insel TR, Hulihan TJ (1995) A gender-specific mechanism for pair bonding: oxytocin and partner preference formation in monogamous voles. Behav Neurosci 109:782-789.

Jones SR, Gainetdinov RR, Wightman RM, Caron MG (1998) Mechanisms of amphetamine action revealed in mice lacking the dopamine transporter. J Neurosci 18:1979-1986.

Kehoe P, Shoemaker WJ, Triano L, Hoffman J, Arons C (1996) Repeated isolation in the neonatal rat produces alterations in behavior and ventral striatal dopamine release in the juvenile after amphetamine challenge. Behav Neurosci 110:1435-1444.

Koob GF, Nestler EJ (1997) The neurobiology of drug addiction. J Neuropsychiatry Clin Neurosci 9:482-497.

Kosten TA, Zhang XY, Kehoe P (2005) Neurochemical and behavioral responses to cocaine in adult male rats with neonatal isolation experience. J Pharmacol Exp Ther 314:661-667. 
Kosten TR, Jalali B, Steidl JH, Kleber HD (1987) Relationship of marital structure and interactions to opiate abuse relapse. Am J Drug Alcohol Abuse 13:387-399.

Leyton M (2010) The neurobiology of desire: dopamine and the regulation of mood and motivational states in humans. In: Pleasures of the brain (Kringelback ML, Berridge KC, eds), pp 222-243. New York: Oxford UP.

Liu Y, Aragona BJ, Young KA, Dietz DM, Kabbaj M, Mazei-Robison M, Nestler EJ, Wang Z (2010) Nucleus accumbens dopamine mediates amphetamine-induced impairment of social bonding in a monogamous rodent species. Proc Natl Acad Sci U S A 107:1217-1222.

Mattson BJ, Williams S, Rosenblatt JS, Morrell JI (2001) Comparison of two positive reinforcing stimuli: pups and cocaine throughout the postpartum period. Behav Neurosci 115:683-694.

McKittrick CR, Abercrombie ED (2007) Catecholamine mapping within nucleus accumbens: differences in basal and amphetamine-stimulated efflux of norepinephrine and dopamine in shell and core. J Neurochem 100:1247-1256.

Nestler EJ (2005) Is there a common molecular pathway for addiction? Nat Neurosci 8:1445-1449.

Panksepp J, Knutson B, Burgdorf J (2002) The role of brain emotional systems in addictions: a neuro-evolutionary perspective and new 'selfreport' animal model. Addiction 97:459-469.

Pitchers KK, Balfour ME, Lehman MN, Richtand NM, Yu L, Coolen LM (2010) Neuroplasticity in the mesolimbic system induced by natural reward and subsequent reward abstinence. Biol Psychiatry 67:872-879.

Robinson TE, Becker JB (1986) Enduring changes in brain and behavior produced by chronic amphetamine administration: a review and evaluation of animal models of amphetamine psychosis. Brain Res 396:157-198.

Robinson TE, Kolb B (1999) Alterations in the morphology of dendrites and dendritic spines in the nucleus accumbens and prefrontal cortex following repeated treatment with amphetamine or cocaine. Eur J Neurosci 11:1598-1604.

Schenk S, Lacelle G, Gorman K, Amit Z (1987) Cocaine self-administration in rats influenced by environmental conditions: implications for the etiology of drug abuse. Neurosci Lett 81:227-231.

Schenk S, Robinson B, Amit Z (1988) Housing conditions fail to affect the intravenous self-administration of amphetamine. Pharmacol Biochem Behav 31:59-62.

Seip KM, Pereira M, Wansaw MP, Reiss JI, Dziopa EI, Morrell JI (2008) Incentive salience of cocaine across the postpartum period of the female rat. Psychopharmacology (Berl) 199:119-130.

Stairs DJ, Klein ED, Bardo MT (2006) Effects of environmental enrichment on extinction and reinstatement of amphetamine self-administration and sucrose-maintained responding. Behav Pharmacol 17:597-604.

Sved AF, Curtis JT (1993) Amino acid neurotransmitters in nucleus tractus solitarius: an in vivo microdialysis study. J Neurochem 61:2089-2098.

Volkow ND, Wang GJ, Fowler JS, Logan J, Gatley SJ, Wong C, Hitzemann R, Pappas NR (1999) Reinforcing effects of psychostimulants in humans are associated with increases in brain dopamine and occupancy of $\mathrm{D}(2)$ receptors. J Pharmacol Exp Ther 291:409-415.

Vungkhanching M, Sher KJ, Jackson KM, Parra GR (2004) Relation of attachment style to family history of alcoholism and alcohol use disorders in early adulthood. Drug Alcohol Depend 75:47-53.

White NM, Chai SC, Hamdani S (2005) Learning the morphine conditioned cue preference: cue configuration determines effects of lesions. Pharmacol Biochem Behav 81:786-796.

Yokel RA, Wise RA (1975) Increased lever pressing for amphetamine after pimozide in rats: implications for a dopamine theory of reward. Science 187:547-549.

Young KA, Gobrogge KL, Wang Z (2011a) The role of mesocorticolimbic dopamine in regulating interactions between drugs of abuse and social behavior. Neurosci Biobehav Rev 35:498-515.

Young KA, Liu Y, Gobrogge KL, Dietz DM, Wang H, Kabbaj M, Wang Z (2011b) Amphetamine alters behavior and mesocorticolimbic dopamine receptor expression in the monogamous female prairie vole. Brain Res 1367:213-222. 